\title{
Exploring Wild Tomato Leaf Extracts in Pesticide Formulations
}

\section{George F Antonious*}

College of Agriculture, Food Science and Sustainable Systems, Division of Environmental Studies, Kentucky State University, Frankfort, Kentucky, USA

\begin{abstract}
Crude extracts from the leaves of the wild tomato, Lycopersicon hirsutum f. glabratum accession PI 134417 possess a pestresistance mechanism in their glandular trichomes (plant hairs) and the exudates they produce due to the presence of a mixture of methyl ketones (MKs) that has insecticidal and acaricidal properties. Type IV and VI glandular trichomes on the leaves of PI 134417 grown under greenhouse conditions were counted. Major volatile oils from glandular leaf trichomes were extracted, purified, and quantified using gas chromatography (GC) and mass spectrometry (GC/MS). The main objectives of this investigation were to: 1) monitor seasonal glandular trichomes density on $\mathrm{PI} 134417$ for mass production of MKs and 2) prepare a simplified formulation of MKs for potential use of PI 134417 leaf extracts, which could become a valuable source of natural products, in plant protection against spider mites and cowpea aphids. Type IV and type VI trichomes were greatest $(\mathrm{P}<0.05)$ in September and October $(97$ and 238 trichomes mm-2), respectively. Quantification of MKs in emulsified extracts of PI 134417 revealed the dominance of 2-tridecanone (the 13-carbon methyl ketone) compared to other MKs (2-undecanone, 2-dodecanone, and 2-pentadecanone) on the leaf surface. Crude leaf extracts of PI 134417 prepared in water caused 33 and $22 \%$ mortality, whereas, water extracts containing $1 \%$ Alkamuls (an organic emulsifier) caused 93 and $82 \%$ mortality of spider mites and cowpea aphis, respectively, $1 \mathrm{~d}$ after exposure.
\end{abstract}

Keywords: Glandular trichomes; Alkamuls; Methyl ketones; Natural insecticides; GC/MSD; Green extraction

\section{Introduction}

There are approximately 500 agrochemical active ingredients manufactured from organic synthesis that account for over $90 \%$ of the total world market. Uttley [1] reported that during the last 15 years, agrochemical innovation has declined and now only 4-8 new Active Ingredients (AIs) are introduced into the market each year. However, there has been a significant increase in the number of new products reaching the market place primarily as a result of mixed products. This indicates that the majority of new patent applications for agrochemical products are not due to discovery of new AIs but for secondary patents resulting from mixing already known synthetic AIs, but prepared in new formulations.

While significant research effort is currently directed toward biological and cultural control strategies against agricultural pests, the application of synthetic pesticides remains an essential activity in many production systems. Pesticide resistance is increasing and the development and registration rate of new pest control chemicals on vegetables (minor crops) is low. Because of the inherent toxicity of most existing synthetic pesticides to non-target organisms and because of their persistence in the environment [2-5], there is increasing pressure on the agricultural industry to find acceptable pest control alternatives. Concerns about pesticide safety usually involve two sides, the environment and the end-user. To protect the environment, the general trend is to use reduced levels of active ingredients. This trend creates a need for pesticide formulations with improved efficacy at low application rates. To protect the end-user, safe formulations that eliminate organic solvent-based formulations are needed. Some liquid formulations, such as emulsifiable concentrates (EC), are harmful not only because of the toxicity of their active ingredients but also because of the toxicity of their organic solvents. These formulations are also coming under more and more regulatory pressure due to their organic solvent content. Plants produce a vast array of volatiles such as monoterpenes and sesquiterpene hydrocarbons that play an important role in plant defense mechanism. The development of efficient natural products with low mammalian toxicity and little or no impact on environmental quality for use against vegetables pests that have gained resistance against many classes of insecticides is needed.

There appear to be no environmental studies conducted with specific reference to the use of methyl ketones (MKs) on vegetables, or any other crops, as organic insecticides and the use of MKs in plant protection have received little attention. Four MKs (2-undecanone, 2-dodecanone, 2-tridecanone, and 2-pentadecanone) were detected in five Lycopersicon hirsutum f. glabratum (Mull) accessions (PI 251304, PI 126449, PI 134417, PI 134418, and LA 407) [6]. 2-tridecanone, the predominant MK in five L. hirsutum accessions analyzed, has shown insecticidal and acaricidal performance against several vegetable insects and spider mites [7]. Research on the wild tomato, L. hirsutum f. glabratum, has demonstrated that their glandular trichomes (plant hairs) and the exudates they produce contribute to insect resistance [6-8]. Such findings on the insecticidal performance of L. hirsutum extracts makes wild tomato an attractive system for study against vegetable insects that have developed resistance to all major classes of modern synthetic insecticides.

The use of natural products for pest control in crop production has been proposed for sustainable agriculture [2]. Wild tomato leaf extracts could be explored as an alternative to synthetic pesticides due to the presence of MKs in their glandular trichomes. MKs (2-undecanone, 2-dodecanone, 2-tridecanone, and 2-pentadecanone) were effective against the tobacco hornworm, Manduca sexta L. and the tobacco budworm, Heliothis virescens Fabricius. 2-tridecanone also was effective against adults of the green peach aphid, Myzus persicae Sulzer and required a significantly lower dose than 2-undecanone [7]. The toxicity of two MKs (2-undecanone and 2-tridecanone), the major constituents of the accessions tested, to adults of the sweet potato whitefly, Bemisia tabaci (Gennadius) and $4^{\text {th }}$ instar larvae of the Colorado potato beetle (CPB), Leptinotarsa decemlineata (Say) determined using no-choice

*Corresponding author: George F. Antonious, Professor, College of Agriculture, Food Science and Sustainable Systems, Division of Environmental Studies, Kentucky State University, 104 Chappell Building, Frankfort, KY 40601-2355, USA Tel: 5025976005; Fax: 5025976763; E-mail: george.antonious@kysu.edu

Received December 29, 2015; Accepted January 10, 2016; Published January 15,2016

Citation: Antonious GF (2016) Exploring Wild Tomato Leaf Extracts in Pesticide Formulations. J Environ Anal Toxicol 6: 347. doi:10.4172/2161-0525.1000347

Copyright: ( 2016 Antonious GF. This is an open-access article distributed under the terms of the Creative Commons Attribution License, which permits unrestricted use, distribution, and reproduction in any medium, provided the original author and source are credited. 
bioassays, revealed that 2 -undecanone caused $80 \%$ mortality of the $4^{\text {th }}$ instar larvae of the $\mathrm{CPB}$ at the highest concentration tested $(100 \mathrm{mg}$ 2-undecanone $\mathrm{mL}^{-1}$ of acetone extract) while, 2-tridecanone caused $72 \%$ mortality of whiteflies at $20 \mathrm{mg} 2$-tridecanone $\mathrm{mL}^{-1}$ of ethanol extract [9]. 2-tridecanone, which has a herbaceous, spicy odor [10], was found toxic to a number of insect species by contact, ingestion, or by vapor action $[11,12]$.

Accordingly, the use of natural plant products for pest control may impart a selective advantage to plants by inhibiting, repulsing, and even killing non-adapted organisms that feed upon, or compete with the plant. Review on the wild tomato, L. hirsutum has indicated that their contribution to insect resistance is due to the presence of volatile compounds on their leaves. The main objectives of this investigation were to: 1) monitor seasonal glandular trichomes densities on PI 134417 for mass production of MKs and 2) prepare a simplified formulation of MKs for potential use of PI 134417 leaf extracts, which could become a valuable source of natural products, in plant protection.

\section{Materials and Methods}

Seeds of Lycopersicon hirsutum f. glabratum Mull PI 134417, wild relatives of tomato, were obtained from the USDA/ARS, Plant Genetic Resources Unit, and Cornell University Geneva, NY, USA and germinated in the laboratory on moistened filter paper in Petri dishes kept in the dark. After germination, seedlings were maintained under fluorescent lighting in the laboratory. At the 6-leaf stage, plants were transported to the greenhouse, transplanted into plastic pots, $20 \mathrm{~cm}$ in diameter containing Pro Mix (Kentucky Garden Supply, Lexington, $\mathrm{KY}$ ) and grown under natural day lighting conditions supplemented with sodium lamps providing additional photosynthetic photon flux of $110 \mu \mathrm{mol} \mathrm{s}^{-1} \mathrm{~m}^{-1}$. Pots, spaced $30 \mathrm{~cm}$ apart, were distributed on the greenhouse benches and the plants were irrigated daily and fertilized twice a month with water containing $200 \mathrm{ppm}$ of an organic fertilizer (Daniels Pinnacle Fertilizer; NPK 3-1-1) obtained from Premium Horticultural Supply, Louisville, KY). No insects were observed on the wild tomato foliage and no insecticides were applied. Average greenhouse temperature and relative humidity were $30 \pm 3.9^{\circ} \mathrm{C}$ and $49.5 \pm 11.8 \%$, respectively.

\section{Trichomes counts and preparation of crude leaf extracts}

Three leaves of similar size, free of visible defects were sampled from equivalent positions below the plant apex (designated as node number below the apical meristem). The second, fourth and sixth pairs of leaves were considered upper, middle and lower leaves of each plant, respectively. One leaflet of each pair was used to obtain adaxial (AD) trichome counts and the corresponding opposite leaflet was used for abaxial $(\mathrm{AB})$ counts. Only type IV and VI glandular trichomes (Figure 1) were counted using a light microscope at magnification of $100 \mathrm{X}$ (10X ocular and 10X objective). Three counts were made for each leaflet surface (within interveinal areas) at the top, near the center, and bottom of each leaflet. Numbers of type IV and type VI trichomes $\mathrm{mm}^{-2}$ were recorded and analyzed statistically using ANOVA [13].

A bulk crude extract of MKs was prepared by soaking $20 \mathrm{~g}$ of wild tomato leaves in $200 \mathrm{~mL}$ of water containing 1\% Alkamuls (a nonionic surfactant from castor oil) obtained from Solvay Rhodia Inc. (Cedar Brook Drive, Cranbury, NJ, USA). After continuous manual shaking the mixture was filtered through a Whatman 934-AH glass microfibre filter of $55 \mathrm{~mm}$ diameter (Fisher Scientific, Pittsburg, PA) and the filtrates were partitioned with $100 \mathrm{~mL}$ of $\mathrm{n}$-hexane in a separatory funnel. The hexane layers were combined and evaporated to dryness using a rotary vacuum evaporator (Buchi Rotavapor Model 461, Switzerland) at $35^{\circ} \mathrm{C}$ followed by a gentle stream of nitrogen gas $\left(\mathrm{N}_{2}\right)$. To purify and determine the concentration of each MKs in the crude extract, the concentrated crude extract was re-dissolved in $10 \mathrm{~mL}$ of $\mathrm{n}$-hexane and applied to the top of a glass chromatographic column $(20 \times 1.1 \mathrm{~cm})$ containing $10 \mathrm{~g}$ alumina grade-II that had been pre-wetted and eluted with $100 \mathrm{~mL}$ of $\mathrm{n}$-hexane. The eluent was evaporated to dryness and reconstituted in $\mathrm{n}$-hexane for GC/MSD injections. Recovery values using fortified alumina columns were 96.0, 92.0, 95.3 and $91.7 \%$ for 2-undecanone, 2-dodecanone, 2-tridecanone, and 2-pentadecanone, respectively. MKs in the wild tomato crude extracts were identified and quantified on a Hewlett-Packard (HP) gas chromatograph (GC), model 5890 equipped with mass selective detector (HP 5971) and a HP 7673 automatic injector. The instrument was auto-tuned with perfluorotributylamine (PFTBA) at $\mathrm{m} / \mathrm{z} 69,219$, and 502 using the total ion mode. The electron impact (EI) mass spectra were carried out using an ionization potential of $70 \mathrm{eV}$. The operating parameters of the GC were as follows: injector and detector temperatures 210 and $275^{\circ} \mathrm{C}$, respectively. Oven temperature was programmed from 70 to $230^{\circ} \mathrm{C}$ at a rate of $10^{\circ} \mathrm{C} \mathrm{min}{ }^{-1}$ with $2 \mathrm{~min}$. initial hold. Injections onto the GC column were made in split-less mode using a 4-mm ID single taper liner with deactivated wool. A $25 \mathrm{~m} \times 0.2 \mathrm{~mm}$ ID capillary column containing $5 \%$ diphenyl and $95 \%$ dimethyl-polysiloxane (HP-5 column) with $0.33 \mu \mathrm{m}$ film thicknesses was used. Carrier gas (He) flow rate was $5.2 \mathrm{~mL} \mathrm{~min}^{-1}$. Quantification was based on average peak areas from three consecutive injections. Retention times of 2-undecanone, 2-dodecanone, 2-tridecanone, and 2-pentadecanone under these conditions were $10.22,12.9,14.23$, and $16.75 \mathrm{~min}$, respectively. Peak areas were determined on a Hewlett Packard (HP) Model 3396 Series II integrator. Quantification was carried out using aliquots of one $\mu \mathrm{L}$ injections of diluted extracts. Area units were compared to external standard solutions of 2-Undecanone (98\% purity) and 2-dodecanone (95\% purity) purchased from Acros Organics Chemical Company (Fair Lawn, NJ, USA), and 2-tridecaone (98\% purity) and 2-pentadecanone (97\% purity) purchased from Alfa Aesar Chemical Company (Shore Road, Heysham, England). Linearity over the range of concentrations was determined using regression analysis. The retention time and mass of each of the MKs isolated from L. hirsutum f. glabratum PI 134417 leaf samples matched those from their standards.

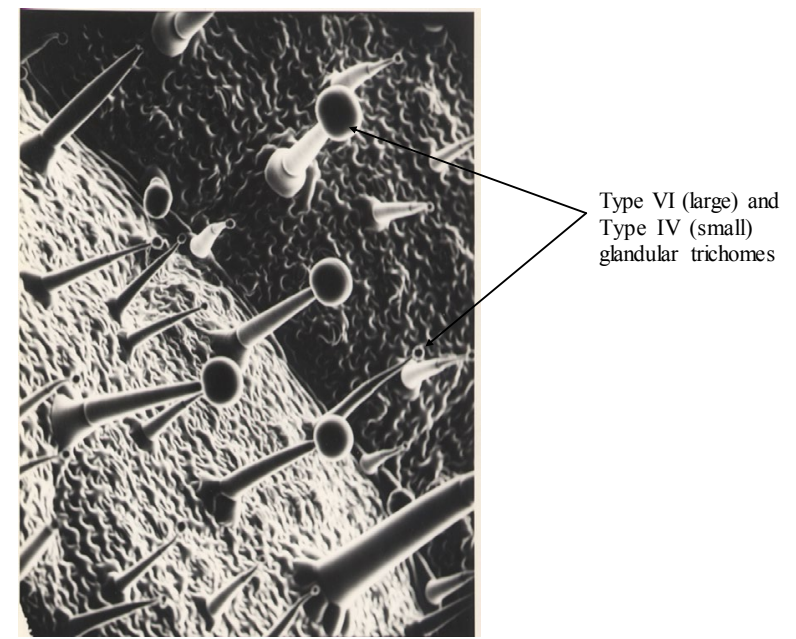

Figure 1: Photomicrograph (400X) of type VI and type IV glandular trichomes on the leaves of Lycopersicon hirsutum f. glanratum obtained using scanning electron microscopy. 


\section{Testing the toxicity of formulated and non-formulated leaf water extracts against spider mites and cowpea aphids}

A two-spotted spider mite, Tetranychus urticae Koch, colony was maintained on green bean plants, Phaseolus vulgaris $\mathrm{L}$., in the laboratory at $50-70 \% \mathrm{RH}, 23.2^{\circ} \mathrm{C}$, with a photoperiod of $24 \mathrm{~h}$ light and were watered daily in the laboratory at the Horticultural Department at University of Kentucky, Lexington, KY, USA. The abaxial (AB) surfaces of bean leaves were sprayed with crude leaf extracts of wild tomato prepared in $1 \%$ Alkamuls (an emulsifier) at different concentrations of leaf extracts $(0,5,10,20,50$ and $100 \%)$ for evaluating spider mite mortality. This stock solution was prepared by soaking PI 134417 leaves in a deionized water solution containing $1 \%$ Alkamuls so that $1 \mathrm{~g}$ of leaves would be equivalent to $4.72 \mathrm{mg}$ of MKs ( $100 \%$ solution), and 0.5 gm of leaves would be equivalent to $2.36 \mathrm{mg}$ of MKs (50\% solution), and $0.25 \mathrm{~g}$ of leaves would be equivalent to $0.944 \mathrm{mg}$ of MKs $(20 \%$ solution), etc. Similarly, another stock solution was prepared from the leaves soaked in water with no-Alkamuls so that one $g$ of leaves would be equivalent to $4.72 \mathrm{mg}$ total MKs as described above. Solutions of leaf extracts were sprayed on bean leaves until moist using a mist sprayer (Meijer, Lexington, KY, USA). The control treatment consisted of misting the leaves with deionized water only. After misting, leaves were allowed to dry under a fume hood. Leaf discs $(1.6 \mathrm{~cm}$ diameter each) were cut and placed on moistened filter paper in a Petri dish (50$9 \mathrm{~mm}$ ) (BD Biosciences, San Jose, CA, USA). Three leaf discs were used for each concentration tested for spider mite mortality. Ten female spider mites were gently placed on each leaf disk using a fine-tipped paint brush. The dishes were labeled and placed in a growth chamber at $28^{\circ} \mathrm{C}$ in complete darkness. Mortality was assessed 4 and $24 \mathrm{~h}$ after treatment by prodding the spider mites gently with a fine-tipped paint brush. If there was no movement, then they were counted as dead. Each concentration was tested on three different days to allow for replication.

For aphid mortality bioassays, sprayed leaf crude extracts were also evaluated in the laboratory for their efficacy against the cowpea aphid, Aphis craccivora Koch. The cowpea aphid colony was maintained on fava beans, Vicia faba under similar laboratory conditions to those for T. urticae. Leaf discs, prepared and treated in a manner similar to those used for spider mites, were used for aphid bioassay. Ten adult aphids were gently placed on the sprayed leaf disks, and the procedure was completed as described for spider mite bioassay employing three replicates, each conducted on a different day. The data were subjected to probit analysis using Polo plus software (LeOra Software, Berkeley, CA, USA) to calculate the percent mortality.

\section{Results and Discussion}

Density of type IV glandular trichomes on the abaxial and adaxial surfaces of leaves of PI 134417 of L. hirsutum f. glabratum varied among the sampling months (Tables 1 and 2, respectively). Type IV trichomes occurred at much higher densities on the abaxial than the adaxial leaf surface. The average total number of type IV on both the abaxial and adaxial leaf surfaces averaged 97 trichomes $\mathrm{mm}^{-2}$ leaf surface during the month of September. Average total type IV trichome densities were significantly lower $(\mathrm{P}>0.05)$ in October $\left(34\right.$ trichomes $\mathrm{mm}^{-2}$ leaf surface) and fluctuated during the other sampling months (Table 3 ). Similarly, density of type VI glandular trichomes on the abaxial and adaxial surfaces of leaves of PI 134417 of L. hirsutum f. glabratum varied among the sampling months (Tables 4 and 5, respectively). Type VI trichomes occurred at much higher densities on the abaxial than the adaxial leaf surface. Average total number of type VI on both the abaxial and adaxial leaf surface averaged 238 and 130 trichomes $\mathrm{mm}^{-2}$ leaf surface during the month of October and November, respectively. Total type VI trichomes densities fluctuated during the other sampling months and were lower in June-September (Table 6).

Gas chromatographic/Mass spectrometric (GC/MS) analyses of standard solutions of four MKs (2-undecanone, 2-dodecanone, 2-tridecanone, and 2-pentadecanone) prepared in $\mathrm{n}$-hexane are presented in Figure 2. Figure 3 indicated that the retention time $(10.22$ $\min )$ and mass of 2-undecanone (170) and its ion fragments in the crude leaf extracts of PI-134417 (upper graph) matched those from Sigma standard and NIST-2 MS library (lower graph). Similarly, results indicated that 2-dodecanone spectral data, which showed a molecular ion peak $(\mathrm{M}+)$ at $\mathrm{m} / \mathrm{z} 184$ along with other characteristic fragment ion peaks (Figure 4) are consistent with the assignment of the molecular formula of 2-dodecanone. 2-tridecanone spectral data showed a molecular ion peak of $\mathrm{m} / \mathrm{z} 198$, along with other ion fragment ions at

\begin{tabular}{|c|c|c|c|c|}
\hline Duncan Grouping & & & Mean & Date \\
\hline & A & & 95.79 & September \\
\hline & A & & & \\
\hline B & A & & 90.833 & May \\
\hline B & A & & & \\
\hline B & A & C & 89.209 & March \\
\hline$B$ & A & $\mathrm{C}$ & & \\
\hline$B$ & A & $\mathrm{C}$ & 86.044 & February \\
\hline$B$ & & $\mathrm{C}$ & & \\
\hline B & & C & 81.418 & April \\
\hline B & & C & & \\
\hline B & & C & 81.416 & August \\
\hline B & & C & & \\
\hline \multirow[t]{6}{*}{$\mathrm{B}$} & & C & 79.126 & July \\
\hline & & $\mathrm{C}$ & & \\
\hline & & C & 76.458 & June \\
\hline & $\mathrm{D}$ & & 20.96 & November \\
\hline & $\mathrm{D}$ & & & \\
\hline & $\mathrm{D}$ & & 16.75 & October \\
\hline
\end{tabular}

Table 1: Average number of type IV glandular trichomes on the abaxial leaf surface of Lycopersicon hirsutum f. glabratum (PI 134417). Statistical comparisons were carried out among sampling months. Values within a column having different letter(s) are significantly different $(P>0.05)$ using Duncan's test.

\begin{tabular}{|c|c|c|}
\hline Duncan Grouping & Mean & Date \\
\hline A & 24.793 & November \\
\hline B & 17.25 & October \\
\hline C & 5.209 & March \\
\hline C & & \\
\hline C & 1.708 & August \\
\hline C & & July \\
\hline C & 1.584 & September \\
\hline C & & \\
\hline C & 0.999 & June \\
\hline C & & April \\
\hline C & 0.584 & \\
\hline C & & February \\
\hline C & 0.541 & \\
\hline C & & May \\
\hline C & 0.209 & \\
\hline C & 0.168 & \\
\hline C & & \\
\hline
\end{tabular}

Table 2: Average number of type IV glandular trichomes on the adaxial leaf surface of Lycopersicon hirsutum f. glabratum (PI 134417). Statistical comparisons were carried out among sampling months. Values within a column having different letter(s) are significantly different $(P>0.05)$ using Duncan's test. 


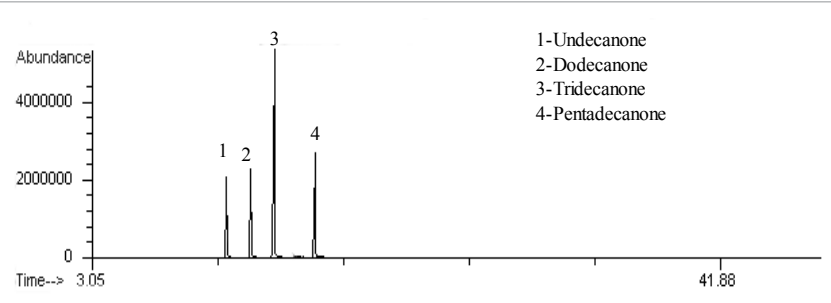

Figure 2: Gas chromatographic (GC) mass spectrometric (MS) chromatograms of four methyl Ketones detected in Lycopersicon hirsutum f. glabratum plant identification (PI) 134417.

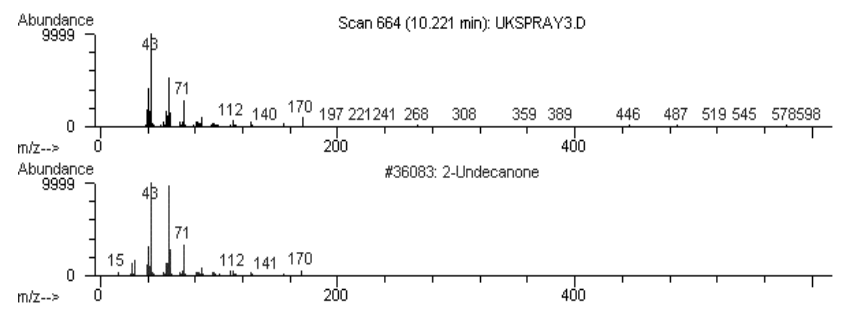

Figure 3: Electron impact mass spectrum of 2-undecanone $\left[\left(\mathrm{CH}_{3}\right)-\left(\mathrm{CH}_{2}\right)_{8}-\mathrm{CO}\right.$ $\mathrm{CH}_{3}$ ] in Lycopersicon hirsutum f. glabratum plant identification (PI) 134417 indicating a molecular weight of 170 along with other characteristic fragment ions.

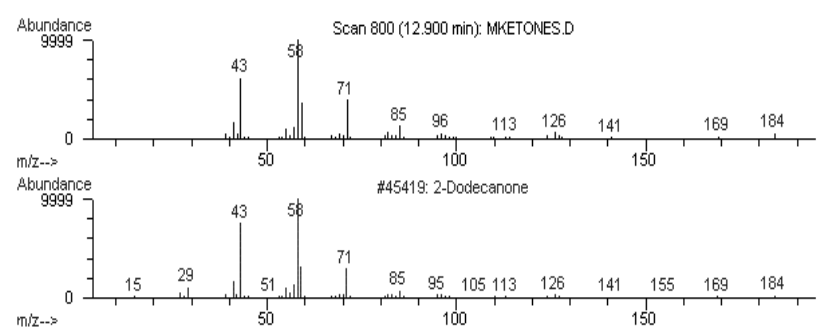

Figure 4: Electron impact mass spectrum of 2-dodecanone $\left[\left(\mathrm{CH}_{3}\right)-\left(\mathrm{CH}_{2}\right)_{9} \mathrm{CO}\right.$ $\mathrm{CH}_{3}$ ] in Lycopersicon hirsutum f. glabratum plant identification (PI) 134417 indicating a molecular weight of 184 along with other characteristic fragment ions.

$96,85,71,58$, and 43 (Figure 5). These ion fragments are also consistent with the Sigma standard and NIST-2 MS library. The presence of 2-pentadecanone in the leaf crude extract of PI 134417, that has a molecular weight of 226 was also confirmed as indicated in Figure 6.

The contents of glandular trichomes on the leaves of PI 134417 were extracted using distilled water. Unexpectedly, all MKs were detected in the GC/MS chromatogram of the water extract prepared from the leaves of Lycopersicon hirsutum f. glabratum of PI 134417 (Figure 7). MKs are not water soluble and require the use of surfactant to enhance MKs water-based extraction. Surprisingly, water extracts of PI 134417 contained the four MKs. Extracting MKs (non-polar active ingredients) using an inorganic solvent (water) is one of the most promising modern green extraction technology. Leaves of PI 134417 were also extracted using a water solution containing 1\% Alkamuls as an emulsifier (Figure 8). This extraction revealed the presence of greater concentrations of MKs when Alkamuls was used compared to the water extracts containing no-Alkamuls (Figure 7).

So far no environmental studies were conducted on the use of MKs as organic pesticide formulation on vegetables or any other crops. Adjuvants (surfactants, wetting agents, emulsifiers, spreaders, stickers, and photostabilizers) are commonly used in agrochemical formulations of pesticides to improve the performance of the active ingredient. Mechanisms of surfactants include stabilization of emulsions and/ or suspensions, increased retention of the active ingredient on plant surfaces, and increased wetting of plant surfaces and subsequent penetration of the active ingredient into plant tissues [14]. Surfactants also reduce droplet surface tension, increasing the contact area between the spray droplet and the leaf surface. These physical properties of surfactants increase droplet contact with the plant foliage and facilitate droplet penetration through the epicuticular plant wax layer. This is because plant cuticles are lipid membranes. They have high sorption capacity for lipophilic solutes like methyl ketones. Surfactants such as triton $\mathrm{H}-100$ (an inert nonionic surfactant) are amphipathic molecules [15]. Many organic compounds which are insoluble in water but soluble in organic solvents may be solubilized in aqueous solution to a certain extent by the presence of surfactants. This characteristic is the primary basis for surfactants widespread use in agrochemical formulations.

The literature review revealed that one of the four MKs, 2-tridecanone, induced elevated levels of cytochrome P-450 isozymes in Heliothis virescens and $H$. zea, rendering their larvae more resistance to some insecticides [16]. However, crude extracts of PI 134417 contain other MKs (2-undecanone, 2-dodecanone, and 2-pentadecanone) that also have insecticidal and acaricidal properties. Because of the presence of more than one active ingredient in PI 134417 crude extracts, insects will have difficulty to resist more than one active ingredient in one formulation. Accordingly, the insecticidal performance of L. hirsutum extracts make wild tomato an attractive system for study against vegetable insects that have developed resistance to all major classes of modern synthetic insecticides.

The two-spotted spider mite, Tetranychus urticae Koch, is a wellknown herbivorous pest of cultivated crops and the cowpea aphids, Aphis craccivora Koch is one of the most widely damaging insects in vegetable production. Alkamuls (an organic emulsifier used in this study) was tested against spider mite and aphid using green bean leaves sprayed with different concentrations of Alkamuls (0-25\%) prepared in water. Results indicated that the toxicity of Alkamuls was significantly greater to spider mites compared to cowpea aphids (Figure 9). Crude water extracts of PI 134417 containing no-Alkamuls and crude extracts prepared in water containing $1 \%$ Alkamuls were tested against the two indicator organisms (spider mite and cowpea aphids). Results indicated that water extracts of PI 134417 exhibited greatest spider mite mortality (15\%) compared to aphid mortality (9.5\%) $4 \mathrm{~h}$ after treatment at the highest concentration tested (Figure 10, upper graph). Similarly, spider mite mortality was greatest (33\%) compared to aphid morality $(22 \%)$ at the same concentration tested $(100 \%$ of crude leaf extracts prepared in water) (Figure 10, lower graph). Results also indicated that crude extracts of PI 134417 leaves prepared in 1\% Alkamuls increased spider mite morality to $92 \%$ and aphid mortality to $76 \%$ at $4 \mathrm{~h}$ after treatment and to $93 \%$ and $82 \%$ at $24 \mathrm{~h}$ after treatment (Figure 11 upper and lower graphs), respectively. Accordingly, a simple preparation of MKs in crude extract of PI 134417 (Figure 12) could be explored by: 1 - collecting $3 \mathrm{~kg}$ of leaves, 2 - mixing the leaves with $6 \mathrm{~L}$ of water containing $60 \mathrm{~mL}$ of $1 \%$ Alkamuls EL-620 (an emulsifier), 3stirring the leaves solution to increase the solubility and extraction of trichomes contents, 4- draining the homogenate using a cheesecloth, and 5-spraying the leaf crude extract on plant surfaces. Analyses of the crude water extract of PI 134417 revealed the presence of $2.1 \mathrm{mg}$ of MKs $\mathrm{g}^{-1}$ of leaves. Whereas, crude extracts prepared in $1 \%$ Alkamuls contained $4.8 \mathrm{mg}$ of $\mathrm{MKs} \mathrm{g}^{-1}$ of leaves. Further studies are needed to test the efficiency and stability of this simple formulation on sprayed plants under field conditions. 


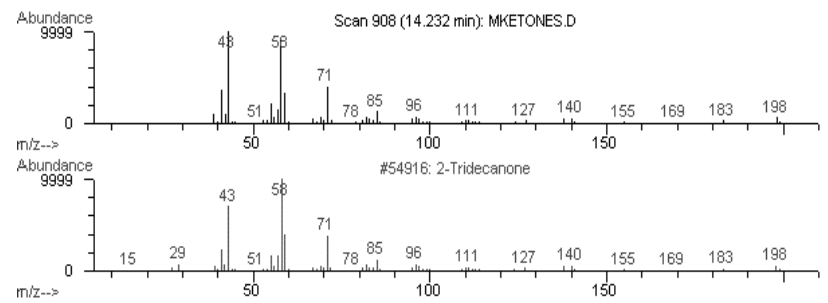

Figure 5: Electron impact mass spectrum of 2-tridecanone $\left[\left(\mathrm{CH}_{3}\right)-\left(\mathrm{CH}_{2}\right)_{10} \mathrm{CO}\right.$ $\mathrm{CH}_{3}$ ] in Lycopersicon hirsutum f. glabratum plant identification (PI) 134417 indicating a molecular weight of 198 along with other characteristic fragment ions.

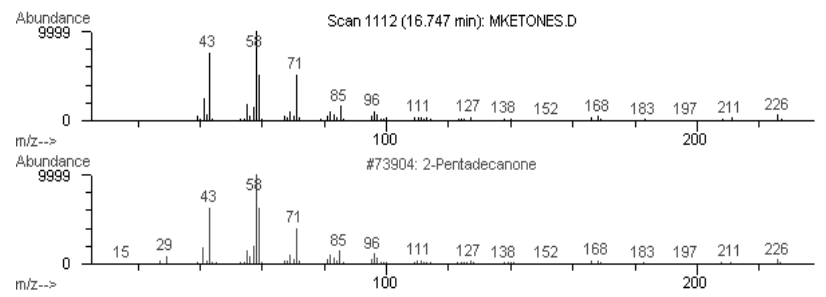

Figure 6: Electron impact mass spectrum of 2-pentadecanone $\left[\left(\mathrm{CH}_{3}\right)-\left(\mathrm{CH}_{2}\right)_{12}\right.$ CO- $\mathrm{CH}_{3}$ in Lycopersicon hirsutum f. glabratum plant identification (PI) 134417 indicating a molecular weight of 226 along with other characteristic fragment ions.

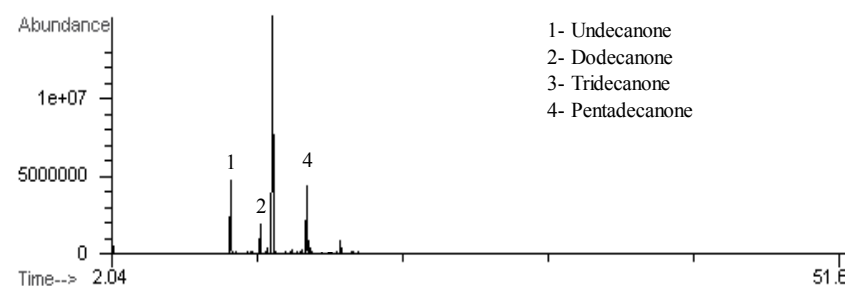

Figure 7: Gas chromatographic (GC) mass spectrometric (MS) chromatogram of water extract prepared from the leaves of Lycopersicon hirsutum f. glabratum plant identification (PI) 134417.

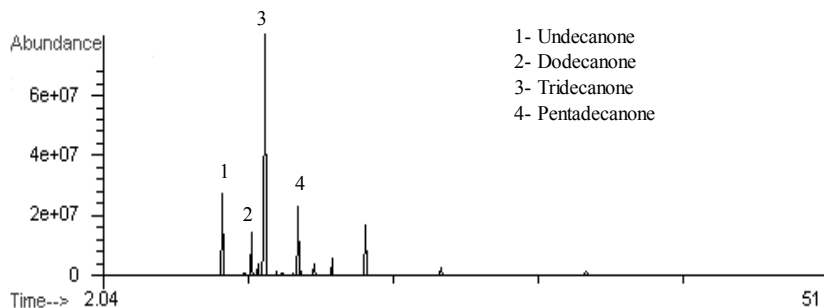

Figure 8: Gas chromatographic (GC) mass spectrometric (MS) chromatogram of water extract containing $1 \%$ Alkamuls prepared from the leaves of Lycopersicon hirsutum f. glabratum plant identification (PI) 134417.

\section{Conclusion}

The wild tomato, Lycopersicon hirsutum f. glabratum Mull, plant identification (PI) 134417 contains anti-arthropod hydrocarbon compounds known as methyl ketones (MKs). Recent trends in extraction techniques have largely focused on finding alternatives that minimize the use of organic solvents. Most organic solvents are flammable, volatile, toxic, and are responsible for environmental pollution and the greenhouse effect [17]. The goal of this investigation was to prepare a simple formulation of MKs using affordable, cheap, and safe solvent.

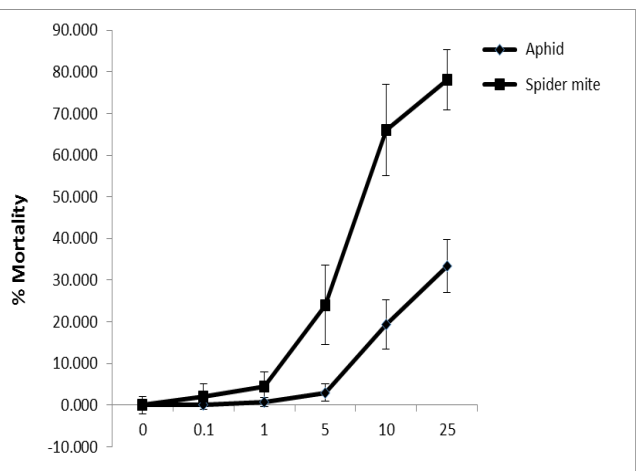

Alkamus Concentration, \%

Figure 9: Percent mortality of spider mite and cowpea aphids $4 \mathrm{~h}$ after treatment with different concentrations of Alkamuls prepared in water.
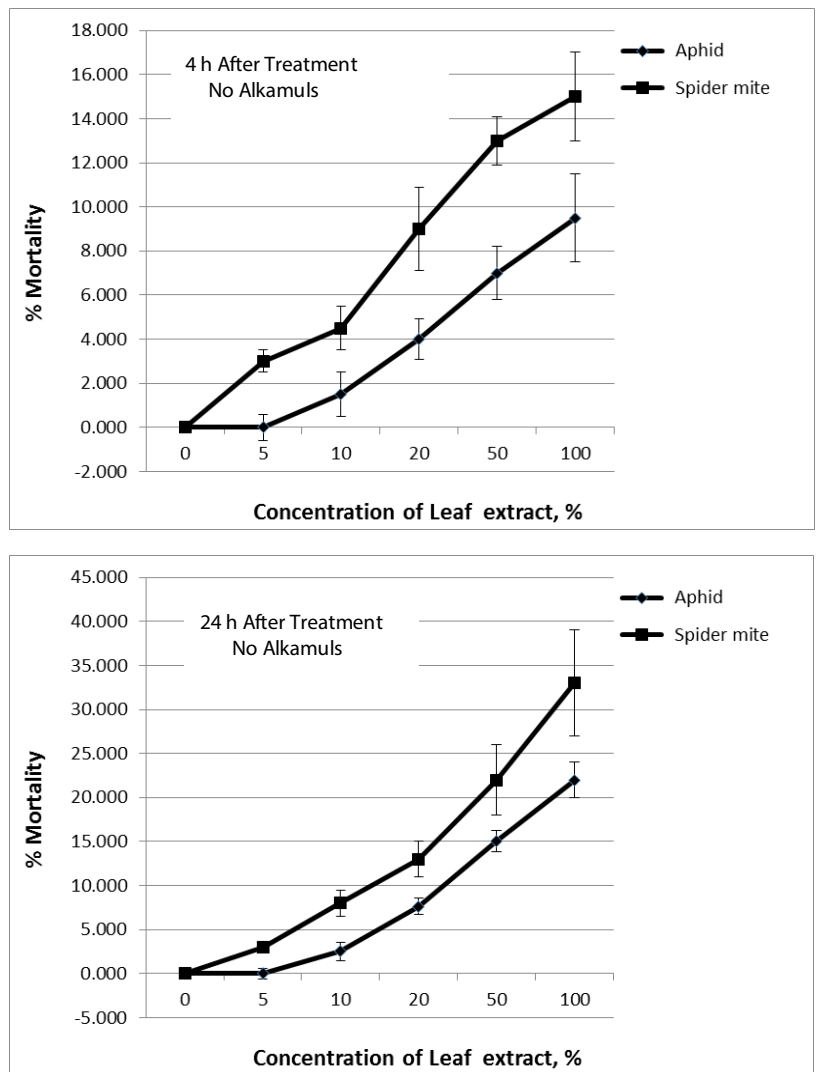

Figure 10: Percent mortality of spider mite and cowpea aphids $4 \mathrm{~h}$ (upper graph) and $24 \mathrm{~h}$ (lower graph) after treatment with crude extracts of PI 134417 prepared in deionozed water (no Alkamuls)

MKs are not water soluble and require the use of surfactant to enhance their water-based extraction. Surprisingly, water extracts of PI 134417 contained MKs (non-polar compounds). Extracting MKs (non-polar active ingredients) using an inorganic solvent (water) is one of the most promising modern green extraction technology. Green extraction reduces energy consumption, allows the use of alternative inorganic solvents and renewable natural resources, and ensures a safe extraction product. Recent trends in extraction techniques have largely focused on finding alternatives that minimize the use of organic solvents. Wild tomato leaves of L. hirsutum f. glabratum, contain phytochemicals such as MKs that could be formulated and explored on a large-scale as a source of biodegradable pest control agents for organic growers. 
Citation: Antonious GF (2016) Exploring Wild Tomato Leaf Extracts in Pesticide Formulations. J Environ Anal Toxicol 6: 347. doi:10.4172/21610525.1000347

Page 6 of 7
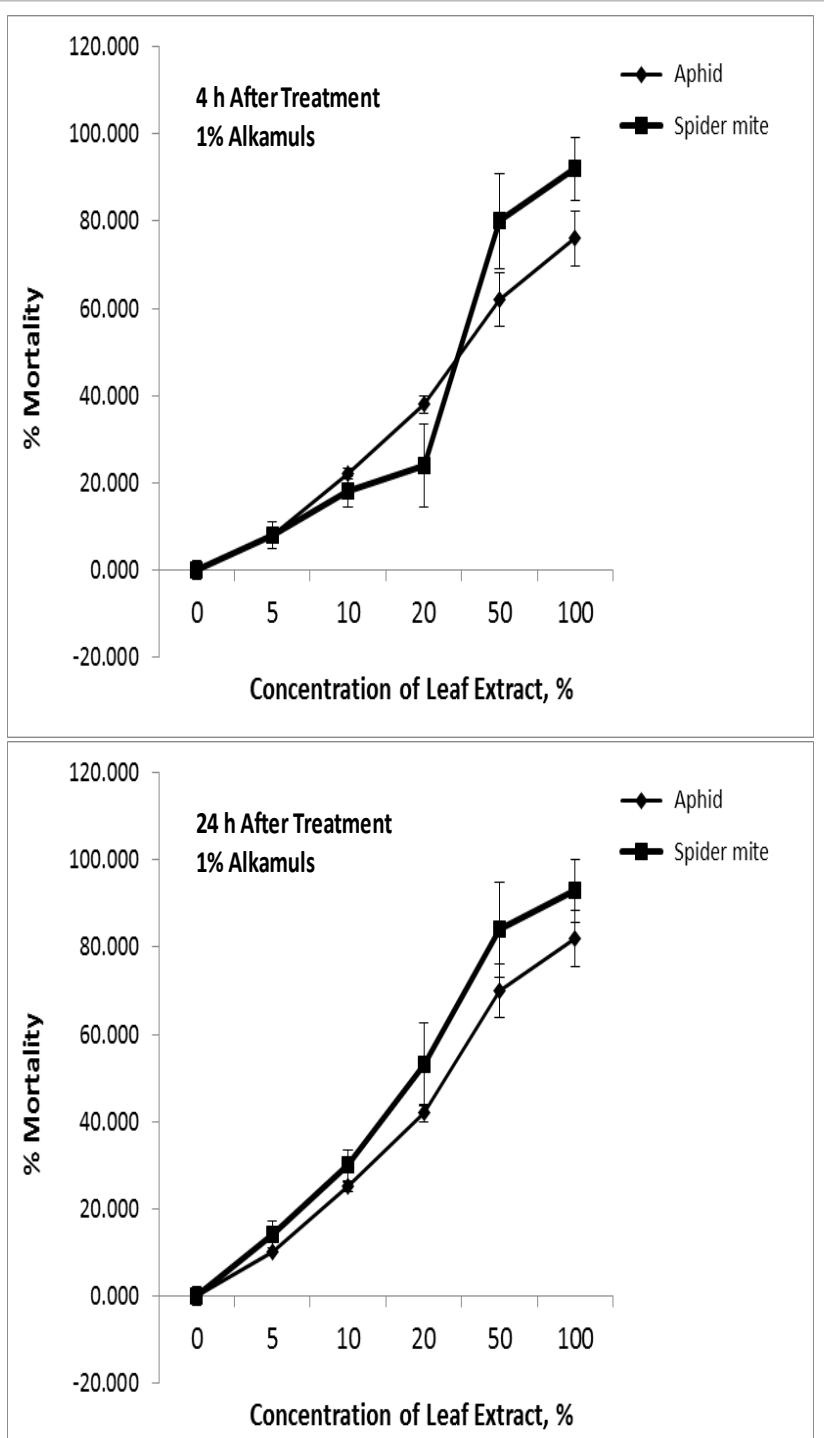

Figure 11: Percent mortality of spider mite and cowpea aphids $4 \mathrm{~h}$ (upper graph) and $24 \mathrm{~h}$ (lower graph) after treatment with crude extracts of PI 134417 prepared in $1 \%$ Alkamuls in water.

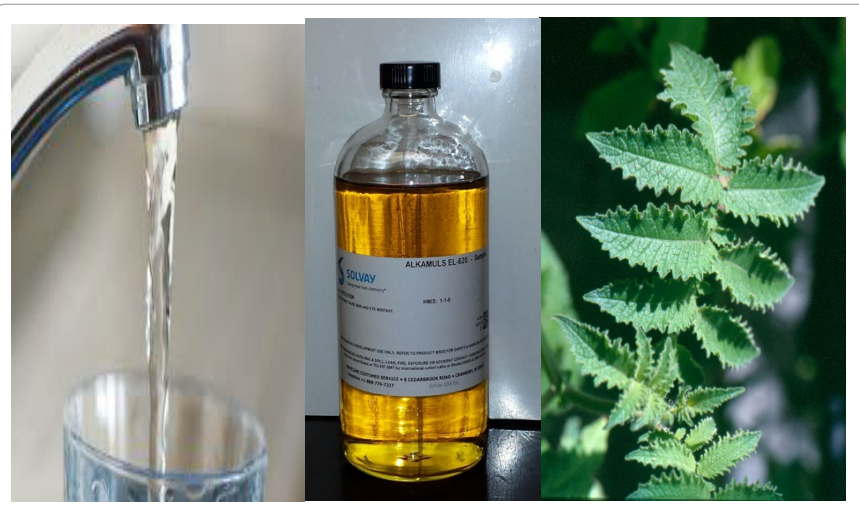

Figure 12: A simplified preparation of Lycopersicon hirsutum f. glabratum (PI 134417) leaf trichomes extraction recipe (mixture of water $+1 \%$ Alkamuls+leaves).

\begin{tabular}{|c|c|c|c|c|}
\hline \multicolumn{3}{|c|}{ Duncan Grouping } & \multirow{2}{*}{$\begin{array}{c}\text { Mean } \\
96.789\end{array}$} & \multirow{2}{*}{$\begin{array}{c}\text { Date } \\
\text { September }\end{array}$} \\
\hline & A & & & \\
\hline & A & & & \\
\hline B & A & & 94.418 & March \\
\hline$B$ & A & & & \\
\hline$B$ & A & C & 91 & May \\
\hline$B$ & A & C & & \\
\hline$B$ & A & C & 86.253 & February \\
\hline$B$ & A & C & & \\
\hline B & A & C & 83.124 & August \\
\hline B & A & C & & \\
\hline B & A & C & 81.959 & April \\
\hline B & & C & & \\
\hline \multirow[t]{6}{*}{ B } & & C & 80.71 & July \\
\hline & & C & & \\
\hline & & C & 77.041 & June \\
\hline & $\mathrm{D}$ & & 45.753 & November \\
\hline & $\mathrm{D}$ & & & \\
\hline & $\mathrm{D}$ & & 34 & October \\
\hline
\end{tabular}

Table 3: Total number of type IV glandular trichomes on the abaxial and adaxial leaf surface of Lycopersicon hirsutum f. glabratum (PI 134417). Statistical comparisons were carried out among sampling months. Values within a column having different letter(s) are significantly different $(\mathrm{P}>0.05)$ using Duncan's test.

\begin{tabular}{|c|c|c|}
\hline Duncan Grouping & Mean & Date \\
\hline A & 237.29 & October \\
\hline B & 127 & November \\
\hline C & 37.58 & June \\
\hline C & & \\
\hline C & 34.54 & August \\
\hline C & & July \\
\hline C & 28.92 & \\
\hline C & & April \\
\hline C & 25.12 & May \\
\hline C & & March \\
\hline C & 24.04 & \\
\hline C & & September \\
\hline C & 23.79 & \\
\hline C & & February \\
\hline C & 23.67 & \\
\hline C & 16.5 & \\
\hline C & & \\
\hline
\end{tabular}

Table 4: Average number of type VI glandular trichomes on the abaxial leaf surface of Lycopersicon hirsutum f. glabratum (PI 134417). Statistical comparisons were carried out among sampling months. Values within a column having different letter(s) are significantly different $(P>0.05)$ using Duncan's test.

\begin{tabular}{|c|c|c|c|}
\hline \multicolumn{2}{|c|}{ Duncan Grouping } & Mean & Date \\
\hline & A & 29.916 & June \\
\hline B & A & & August \\
\hline B & A & 27.041 & \\
\hline B & & & July \\
\hline & C & 23.291 & April \\
\hline D & C & & \\
\hline D & C & 21.125 & March \\
\hline D & C & & \\
\hline D & C & 21 & May \\
\hline D & C & & September \\
\hline D & C & 20.958 & \\
\hline D & & & \\
\hline
\end{tabular}


Citation: Antonious GF (2016) Exploring Wild Tomato Leaf Extracts in Pesticide Formulations. J Environ Anal Toxicol 6: 347. doi:10.4172/21610525.1000347

\begin{tabular}{|c|c|c|c|}
\hline D & & & \\
\hline D & & 15.293 & February \\
\hline & E & 3.334 & November \\
\hline & E & 1.083 & October \\
\hline
\end{tabular}

Table 5: Average number of type VI glandular trichomes on the adaxial leaf surface of Lycopersicon hirsutum f. glabratum (PI 134417). Statistical comparisons were carried out among sampling months. Values within a column having different letter(s) are significantly different $(P>0.05)$ using Duncan's test.

\begin{tabular}{|c|c|c|}
\hline Duncan Grouping & Mean & Date \\
\hline A & 238.37 & October \\
\hline B & 130.33 & November \\
\hline C & 67.5 & June \\
\hline C & & \\
\hline C & 61.58 & August \\
\hline C & & July \\
\hline C & 52.21 & \\
\hline C & & April \\
\hline C & 46.25 & May \\
\hline C & & \\
\hline C & 45 & March \\
\hline C & & \\
\hline C & 44.79 & September \\
\hline C & & February \\
\hline C & 40.5 & \\
\hline C & 31.79 & \\
\hline C & & \\
\hline
\end{tabular}

Table 6: Total number of type IV glandular trichomes on the abaxial and adaxial leaf surface of Lycopersicon hirsutum f. glabratum (PI 134417). Statistical comparisons were carried out among sampling months. Values within a column having different letter(s) are significantly different $(P>0.05)$ using Duncan's test.

\section{Acknowledgements}

I thank Steven Diver for his kind help in maintaining the greenhouse wild tomato plants. This investigation was supported by a Capacity Building Grant from the United States of Agriculture/National Institute of Food and Agriculture (USDA NIFA) to Kentucky State University under agreement No. KYX 2011-38821-30972.

\section{References}

1. Uttley N (2015) Mixtures, formulations dominate new products. Farm Chemical International, Advancing Global Crop Protection and Plant Health Technology.

2. Antonious GF (2015) Decontamination of pesticide residues for sustainable agriculture. JSM Environ Sci and Ecology 3: 1014.
3. Antonious GF (2015) Reducing herbicides in agricultural runoff and seepage water. In: Herbicides, Physiology of Action and Safety. Andrew Price, Linda Sarunaite and Jessica Kelton (Eds). Intech, Rijeka, Croatia.

4. Antonious GF (2004) Trifluralin residues in runoff and infiltration water from tomato production. Bull Environ Contam Toxicol 72: 962-969.

5. Antonious GF (2003) Soil infiltration by pesticides. In: Encyclopedia of Pest Management. Pimentel D (ed) Marcel Dekker Inc., New York 3: 1-4.

6. Antonious GF (2001) Production and quantification of methyl ketones in wild tomato accessions. J Environ Sci Health B 36: 835-848.

7. Antonious GF, Dahlman DL, Hawkins LM (2003) Insecticidal and acaricidal performance of methyl ketones in wild tomato leaves. Bull Environ Contam Toxicol 71: 400-407.

8. Antonious GF, Kochhar TS (2003) Zingiberene and curcumene in wild tomato J Environ Sci Health B 38: 489-500.

9. Antonious GF, Kochhar TS, Simmons AM (2005) Natural products: seasonal variation in trichome counts and contents in Lycopersicum hirsutum f. glabratum. J Environ Sci Health B 40: 619-631.

10. Vernin G, Vernin C, Pieribattesti JC, Roque C (1998) Analysis of volatile compounds of Psidium cattleianum Sabine fruit from Reunion Island. J Essential Oil Res 10: 353-362.

11. Kennedy GG, Farrar RR, Barbour JD (1994) Field evaluation of insect resistance in a wild tomato and its effects on insect parasitoids. Entomol Exp Appl 71: 211-226.

12. Muigai SG, Schuster DJ, Snyder JC, Scott, JW, Bassett MJ, et al. (2002) Mechanisms of resistance in Lycopersicon germplasm to the whitefly Bemisia argentifolii. Phytoparasitica 30: 347-360.

13. SAS Institute (2003) SAS/STAT Guide, Release 0.03 Edition, SAS Inc., SAS Campus Drive, Cary, NC 27513, USA.

14. Shafer WE, Bukovac MJ (1989) Effects of triton (-100 on sorption of 2-(1-naphthyl) acetic acid by tomato fruit cuticles. J Agric Food Chem 37: 486492.

15. Shafer WE, Bukovac MJ (1987) Studies on Octylphenoxy Surfactants : III. Sorption of Triton X-100 by Isolated Tomato Fruit Cuticles. Plant Physiol 85: 965-970.

16. Kennedy GG, Farrar Jr, Riskallah MR (1987) Induced tolerance of neonate Heliothis zea to host plant allelochemicals and carbaryl following incubation of eggs on foliage of Lycopersicon. hirsutum f. glabratum. Oecologia 73: 615-620.

17. Chemat F, Vian MA, Cravotto G (2012) Green extraction of natural products: concept and principles. Int J Mol Sci 13: 8615-8627. 